\title{
Analyse économique de la production artisanale du chakpalo au Bénin : aspects sociaux et facteurs déterminants de la rentabilité financière de l'activité
}

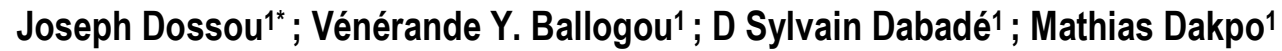 \\ 1 : Département de Nutrition et Sciences Alimentaires, Faculté des Sciences Agronomiques, Université d'Abomey- \\ Calavi, 01 BP 526 Cotonou. Bénin \\ *: Auteur correspondant : E-mail : jokdossou@yahoo.fr ; Tél. :(+229)97086266.
}

Original submitted in on 30th December 2014. Published online at www.m.elewa.org on 31st March 2015 http://dx.doi.org/10.4314/jab.v87i1.7

\section{RÉSUMÉ}

Objectif : la présente étude vise à analyser la rentabilité économique de la production du chakpalo à travers un diagnostic approfondi des charges engagées et une évaluation de la marge brute de production tenant compte des capacités moyennes des différentes catégories de productrices.

Méthodologie et Résultats : l'analyse économique de la production artisanale de chakpalo a été réalisée sur la base des données techniques collectées sur l'activité à travers une enquête diagnostic dans les communes de Dassa-Zoumé et de Glazoué, reconnues comme localités d'origine de cette bière de sorgho au centre du Bénin. Un recensement global des productrices de chakpalo réalisé au préalable dans ces communes a permis d'identifier 459 productrices reparties en trois catégories (petites, moyennes et grandes productrices) et dont 50 ont été sélectionnées pour l'enquête fine. II résulte de cette enquête que la production du chakpalo est une activité séculaire, exclusivement féminine et pratiquée uniquement par les femmes d'ethnie Idaatcha. Plus de $75 \%$ des productrices transforment en moyenne $21,84 \mathrm{~kg}$ de sorgho donnant environ 45 litres de chakpalo par semaine. Sur la base de cette capacité moyenne de production, le résultat d'exploitation obtenu a une valeur négative de -1246,14 FCFA, traduisant ainsi la non rentabilité de l'activité. Le coût de la main d'œuvre ainsi que celui du combustible restent les facteurs déterminants du résultat d'exploitation puisqu'ils atteignent $50 \%$ du total des charges variables. Le seuil de rentabilité correspond à une capacité de $48 \mathrm{~kg}$ de sorgho et il faut transformer environ $50 \mathrm{~kg}$ pour espérer une marge brute positive de 395,45 FCFA.

Conclusion et application : si l'activité se maintient malgré un résultat aussi décevant, c'est à cause de sa portée non financière, puisqu'elle conforte le statut social de la productrice et assure d'importantes fonctions sociocommunautaires en fournissant de la boisson locale appropriée pour les cérémonies rituelles et les fêtes traditionnelles et entretient des marchés de vente bien ancrés dans la société.

Mots clés : bière traditionnelle, analyse économique, seuil de rentabilité. 


\section{Dossou et al. J. Appl. Biosci. Analyse économique de la production artisanale du chakpalo au Bénin : aspects sociaux et facteurs déterminants de la rentabilité financière de l'activité}

Economic study of traditional production of chakpalo in Benin: social aspects and factors determining the financial profitability of the production

\section{Abstract}

Objectives: this study focused on profitability of chakpalo production through a detailed diagnosis of engaged charges and an assessment of the processing gross profit margin considering the average capacity of different producers' class.

Methodology and Results: economic study of traditional chakpalo production was carried out on the basis data collected through a survey in chakpalo areas of origin (central part of Benin).. A previous census in the chakpalo production areas of the municipalities of Dassa-Zoumé and Glazoué identified 459 producers, split in three groups namely small, middle and big producers. A sample of 50 producers was selected from the 459 producers for a deepened survey. Results showed that the production of chakpalo was an exclusively women devoted secular activity. Only women belonging to the ethnic group Idaatcha practiced this activity and more than $75 \%$ of them had an average production capacity of $21.84 \mathrm{~kg}$ of raw sorghum corresponding to 45 litters of chakpalo per week. On the basis of this middle production's capacity, the profitability was estimated to be a negative value of -1246.14 FCFA, meaning that the activity is not profitable. The profitability of this activity depends mainly on the costs of labor and combustible which reached $50 \%$ of total variable costs. The break-even is founded by a capacity of $48 \mathrm{~kg}$ of raw sorghum and a positive profitability of 395.45 FCFA only by a capacity of $50 \mathrm{~kg}$ of sorghum.

Conclusion and application: this activity has persisted until today because it fortifies the social status of the producing women and plays an important social role consisting in the appropriate beverage for ritual ceremonies, traditional festivals and maintains its own market in the society.

Keys words: traditional beer, economic analysis, break-even point.

\section{INTRODUCTION}

Dans la plupart des pays africains et en particulier au Bénin, où le secteur industriel est peu développé, une importante partie des produits agricoles est transformée grâce à l'artisanat alimentaire. Ce secteur d'activités de transformation emploie surtout la population féminine, tant en milieu urbain que rural. La production commerciale du chakpalo en est un exemple ; elle constitue l'une des principales activités de transformation exercées par les femmes du département des Collines, notamment des communes de Glazoué et de Dassa-zoumè au Bénin. Le chakpalo est une boisson fermentée, préparée selon un procédé traditionnel qui comporte principalement une étape de fermentation spontanée d'un moût (Okoumassoun, 1984 ; Atègbo, 1985 ; Nago, 1989). Dossou et al. (2011) et Ballogou et al. (2011) ont décrit, comme principales étapes de la production du chakpalo, le maltage de la céréale (sorgho, maïs et/ou le mélange du sorgho et du maïs), le brasage du malt et la fermentation du moût. Cette activité revêt une importance socio-économique considérable, en particulier pour les femmes des localités concernées. Kayodé et al. (2007) ont attribué ce caractère socio-économique remarquable de la production de la bière de sorgho à son utilisation abondante au cours des cérémonies traditionnelles. De plus, elle constitue une importante source de revenus pour les femmes qui la produisent à l'échelle locale, en utilisant la technologie traditionnelle. Plus récemment, Konfo et al. (2014) ont rapporté que le chakpalo est une boisson très appréciée par les populations béninoises, tandis que Kayodé et al. (2007) ont estimé qu'elle est souvent attachée aux traditions d'hospitalité et de convivialité des familles. Cependant, la production du chakpalo est une activité fastidieuse pour les femmes qui la pratiquent. Selon la FAO (1985), les deux tiers environ des heures de travail effectuées dans le monde sont le fait des femmes et essentiellement des femmes vivant dans les zones rurales. Dans les pays en voie de développement, ces femmes transforment les produits alimentaires sans l'aide d'instruments modernes, soit par routine, soit par 


\section{Dossou et al. J. Appl. Biosci. Analyse économique de la production artisanale du chakpalo au Bénin : aspects sociaux et facteurs déterminants de la rentabilité financière de l'activité}

l'obligation de préserver les matières premières contre les pertes post-récolte ou par le souci de conserver et de transmettre la technologie utilisée à la descendance. Dans la plupart des cas, les recettes brutes tirées de ces activités informelles constituent la seule motivation des productrices puisque le niveau de production est souvent en dessous du seuil de rentabilité. Certes, il manque des données statistiques pour confirmer ces constats pour les productrices de chakpalo du Bénin; mais la pénibilité et la longue durée (en moyenne 10 jours par cycle) de production de cette boisson constituent des difficultés supplémentaires pour ces productrices et en même temps un goulot d'étranglement qui limite la diffusion de la technologie. Les équipements

\section{MATÉRIEL ET MÉTHODES}

Cadre de l'étude : la production du chakpalo est une spécialité des peuples Nagot installés principalement dans les communes de Glazoué et de Dassa-Zoumè dans la région centrale du Bénin. Cette localité a été donc identifiée comme la zone de la présente étude. Ces deux communes sont situées dans le département des Collines et sont majoritairement peuplées des Idaatcha, qui constituent, selon la tradition orale, l'ethnie dépositaire de la technologie originelle de production du chakpalo au Bénin. L'aire géographique occupée par ce peuple s'étend de la rive gauche du fleuve Zou jusqu'au fleuve Ouémé, à la limite sud des communes de Savè, Ouèssè et Bantè. La commune de Glazoué dispose d'un marché international, de 10 marchés quotidiens et 11 marchés périodiques; tandis que celle de Dassa-Zoumé compte 3 marchés quotidiens et 10 marchés périodiques. Ces marchés jouent un important rôle dans la vie économique de ces communes et en particulier dans la commercialisation du chakpalo.

Échantillonnage: un recensement global des productrices de chakpalo a été d'abord effectué dans les différents villages de la commune de Dassa-Zoumè et de Glazoué. Ainsi, il a été dénombré environ 125 productrices dans la commune de Dassa-Zoumè et 334 productrices dans la commune de Glazoué, soit environ 459 productrices dans les deux communes. Ensuite un échantillon restreint a été identifié pour l'enquête fine. Les villages choisis constituent les zones où l'activité de production du chakpalo est plus importante. 50 productrices ont été retenues dans les deux communes utilisés sont rudimentaires, affectant ainsi la qualité de la boisson. Les gourdes et les calebasses servant à conditionner le chakpalo sont fragiles, deviennent rares, plus coûteuses et pourtant ne répondent pas aux exigences des consommateurs modernes. Mais de toutes ces contraintes, c'est le manque de données chiffrées sur la valeur ajoutée, en particulier sur la marge bénéficiaire de cette activité qui motive la présente étude dont l'objectif principal est d'analyser la rentabilité économique de la production du chakpalo. Plus spécifiquement, elle fait un diagnostic approfondi des charges engagées et évalue la marge brute de la production du chakpalo, en tenant compte des capacités moyennes des différentes catégories de productrices.

et réparties en fonction de la prépondérance de l'activité dans chacune des localités ciblées; il a été choisi, par ordre d'importance, dans la commune de Glazoué les villages Yawa, Orokoto, Sokponta, Gomé et le marché de Glazoué, et dans la commune de Dassa-Zoumè, Okéméré, Lèma, Kèrè et les quartiers Ayédèro et Essèkpa dans la ville de Dassa (Tableau 1).

Méthode d'enquête et types de données collectées : les données ont été collectées à l'aide de la Méthode Accélérée de Recherche Participative (MARP), comportant des entretiens structurés, semistructurés et non structurés et des observations participantes. Les entretiens semis-structurés et non structurés ont été utilisés pendant toute la durée de l'enquête, mais surtout lors de la phase exploratoire. Cette première étape a été réalisée dans quelques villages, à savoir, Orokoto, Gomé, Sokponta, Okéméré, dans les quartiers de villes de Dassa et dans le marché de Glazoué. Les Centres Communaux de Promotion Agricole et les mairies de Dassa-Zoumé et de Glazoué ont été contactés pour vérifier et compléter les informations obtenues. Les entretiens structurés ont été réalisés à l'aide d'un questionnaire administré aux productrices individuelles impliquées dans l'enquête. Des observations participantes nous ont permis de décrire la technologie de fabrication du chakpalo, de quantifier les matières premières à l'aide d'une balance de $25 \mathrm{~kg}$ de portée et d'évaluer la durée des opérations à l'aide d'un chronomètre. Cette enquête a permis de collecter des données plus spécifiques, notamment socioculturelles des productrices de chakpalo (âge, 


\section{Dossou et al. J. Appl. Biosci. Analyse économique de la production artisanale du chakpalo au Bénin : aspects sociaux et facteurs déterminants de la rentabilité financière de l'activité}

niveau d'instruction, ethnie, etc.), les équipements utilisés pour la fabrication du chakpalo et leurs durées d'utilisation respectives, le type de main-d'œuvre utilisée ainsi que les différents coûts liés à la production et le prix de vente du chakpalo.

Tableau 1 : Répartition géographique des enquêtées

\begin{tabular}{llcc}
\hline Communes & Localités & Nombre d'enquêtées & Pourcentage (\%) \\
\hline Glazoué & Marché Glazoué & 3 & 6 \\
& Sokponta & 6 & 12 \\
& Orokoto & 11 & 22 \\
& Yawa & 15 & 30 \\
\multirow{5}{*}{ Dassa-zoumè } & Gomé & 2 & 4 \\
& Lèma & 2 & 4 \\
& Essekpa & 2 & 4 \\
& Okéméré & 6 & 12 \\
& Kèrè & 2 & 4 \\
& Ayedèro & 1 & 2 \\
\hline Total & & 50 & 100 \\
\hline
\end{tabular}

Amortissement des équipements : les charges fixes se résument ici aux amortissements des équipements utilisés pour la production du chakpalo. Pour chaque équipement, il a été estimé le nombre moyen, la durée de vie, le prix unitaire et la durée de son utilisation par cycle de production. L'annuité $\left(A_{\text {mi }}\right)$ par type d'équipement a été obtenue en appliquant la formule : $A_{m i}=\left(N_{i} \times P_{u i} / D_{i}\right) \times(p / 100)$, où $N_{i}$ est le nombre de cet équipement, $P_{u i}$, le prix unitaire et $D_{i}$ la durée de vie de l'équipement et $p$, la proportion de temps d'utilisation de l'équipement par cycle de production. On a supposé que le matériel de production est utilisé en moyenne une fois par semaine ce qui équivaut à 52 utilisations par an. Le prix de revient d'une utilisation a été obtenu en faisant le rapport du prix d'achat par le nombre total d'utilisation possible du matériel.

Estimation de la main-d'œuvre : les productrices de chakpalo utilisent uniquement la main-d'œuvre familiale. Mais il paraît indispensable d'estimer la maind'œuvre salariée pour mieux apprécier la rentabilité financière de l'activité. Le nombre de personnes intervenant pour chaque opération, la durée du travail et les rémunérations appliquées dans la zone d'étude sont connus grâce à l'enquête. La main-d'œuvre a été ensuite évaluée par la méthode de Fabre (1994) qui propose la formule suivante pour le calcul de l'effectif total $(\mathrm{ET})$ des productrices en équivalent-homme :

$\mathrm{ET}=$ nombre d'hommes $+0,75 \mathrm{x}$ (nombre de femmes) $+0,5 \times$ (nombre d'enfants de 6 à 14 ans).
Mais pour tenir compte du fait que l'activité est essentiellement féminine, le coefficient de 0,75 appliqué aux femmes a été arrondi à 1 , modifiant la formule de Fabre comme suit :

$\mathrm{ET}=$ nombre d'hommes + nombre de femmes $+0,5 \mathrm{x}$ (nombre d'enfants de 6 à 14 ans).

Pour la conversion en femme-jour (F/J), nous avons multiplié ET par la durée totale (TD) des opérations estimée en heure divisée par 8. La formule peut s'écrire : $E T_{h . j}=E T x(T D / 8)$.

Coût de l'énergie de cuisson : le prix de revient $C$ de l'énergie consommée est calculé selon la formule proposée par Dossou et al,, (2005) comme suit :

$$
c=r \times t \times q \times p
$$

où $r$ est le rendement de transformation ; $t$ la quantité de sorgho transformée ; q la quantité de combustible consommée et $p$, le prix unitaire du combustible.

Calcul des résultats économiques: la notion de rentabilité traduit ici la marge brute positive donnée par la différence entre les charges et les recettes. Selon Fabre (1994), le résultat brut d'exploitation exprime le gain (ou la perte) économique d'argent une fois acquittées toutes les charges d'exploitation courantes. Le coût de production et les résultats d'exploitation sont calculés à partir des données collectées au cours de l'enquête et intégrés dans le Tableau 4 du compte d'exploitation. 
Tableau 2: Charges fixes pour la production du chakpalo

\begin{tabular}{lccccc}
\hline Matériel & $\begin{array}{c}\text { Quantité } \\
\mathbf{N}_{\mathbf{i}}\end{array}$ & $\begin{array}{c}\text { Coût d'achat } \mathbf{P}_{\text {ui }} \\
\text { (FCFA) }\end{array}$ & $\begin{array}{c}\text { Durée de vie } \\
\mathbf{D}_{\mathbf{i}}(\mathbf{a n})\end{array}$ & $\begin{array}{c}\text { Nombre } \\
\text { d'utilisations } \mathbf{p}\end{array}$ & $\begin{array}{c}\text { Charges } \\
\text { d'amortissement } \\
\text { (FCFA) }\end{array}$ \\
\hline Marmite & 02 & 30000 & 10 & 520 & 57,69 \\
Foyer à trois pierres & 01 & 2500 & 01 & 52 & 48,07 \\
Bassines & 03 & 15000 & 01 & 52 & 288,46 \\
Spatule & 01 & 500 & 01 & 52 & 9,61 \\
Panier & 02 & 1000 & 01 & 52 & 19,23 \\
Jarres & 02 & 10000 & 05 & 260 & 38,46 \\
Gourdes & 03 & 12000 & 01 & 52 & 423,06 \\
Calebasses & 30 & 2500 & 01 & 52 & 48,07 \\
Bâche & 01 & 2000 & 01 & 52 & 38,46 \\
Hangars & 01 & 40000 & 05 & 260 & 153,84 \\
\hline & \multicolumn{7}{c}{} \\
\hline
\end{tabular}

Méthode d'analyse des données: A l'issue de l'enquête, les données collectées ont été saisies et traitées dans le logiciel Excel puis les moyennes ont été calculées. L'analyse financière a été réalisée grâce

\section{RÉSULTATS ET DISCUSSION}

Technologie de production du chakpalo : la matière première utilisée par les productrices enquêtées est le sorgho rouge. Pour Kayodé et al. (2005) et Osseyi et al. (2011), le chakpalo est produit en utilisant le malt de sorgho rouge ou marron (Kayodé et al., 2005 ; Osseyi et al., 2011). La production du chakpalo regroupe principalement les étapes du maltage, du brassage et de la fermentation. L'ensemble du procédé est décrit comme suit.

Le maltage est conduit en trois étapes, à savoir, le trempage, la germination et le séchage. Les grains de sorgho sont vannés, triés et lavés à grande eau pour éliminer les déchets ; le trempage a lieu dans des bassines à la température ambiante pendant $24 \mathrm{~h}$ au maximum. Après le détrempage, les grains sont lavés puis égouttés dans un panier ; ils sont ensuite gardés dans le dispositif d'égouttage et recouverts d'une toile plastique noire pendant environ 10 à $12 \mathrm{~h}$ de prégermination; cette étape permet une amorce de la levée des pousses. La germination proprement dite a été réalisée par étalement du sorgho en couche de 3 à $4 \mathrm{~cm}$ d'épaisseur pendant 3 jours sur des plateaux en plastiques perforés. Les grains mis en germination sont recouverts d'un sac de jute et arrosés toutes les $12 \mathrm{~h}$ pendant $72 \mathrm{~h}$ de germination. On obtient le malt vert. au calcul de l'amortissement, des charges variables (y compris la main-d'œuvre), du coût de l'énergie de cuisson et le résultat d'exploitation.

Après la germination, les grains sont étalés à l'ombre et laissés pendant $24 \mathrm{~h}$ au moins pour permettre aux enzymes d'hydrolyser le résidu d'amidon contenu dans les graines et de libérer les sucres fermentescibles, indispensables au brassage de la bière. Cette opération, appelée mûrissement, rend les enzymes actives mais inhibe le développement des organes végétatifs de la graine (feuilles et racines) surtout les feuilles qui apparaissent après $72 \mathrm{~h}$ de germination. A la fin de cette étape de mûrissement, le malt vert est séché au soleil pendant 24 à $48 \mathrm{~h}$. Le maltage a également pour effet d'augmenter la solubilité dans l'eau des protéines, la teneur en lysine, en méthionine, en sucres solubles et la disponibilité du fer, du calcium, du phosphore (Bhise et al., 1988 ; Traoré et al., 2004). Les grains maltés changent de couleur, le gout et les arômes caractéristiques se développent.

Le brassage effectué au cours de nos observations participantes est le même que celui décrit par les productrices de chakpalo ; il comporte la mouture du malt, la préparation de la maiche, la saccharification et les différentes étapes de cuisson. Le malt est grossièrement moulu dans un moulin à maïs. La farine obtenue est délayée dans de l'eau dans une proportion d'environ un $\mathrm{kg}$ de farine pour trois litres d'eau ; ce 


\section{Dossou et al. J. Appl. Biosci. Analyse économique de la production artisanale du chakpalo au Bénin : aspects sociaux et facteurs déterminants de la rentabilité financière de l'activité}

mélange, appelé maiche, est remué, homogénéisé et laissé décanter pendant environ une heure à la température ambiante. Le $\mathrm{pH}$ de la maiche est en moyenne de 5,2. Le surnageant est ensuite délicatement séparé du dépôt. Cette étape permet de solubiliser les sucres et d'extraire les enzymes du malt indispensables à la saccharification de l'amidon. II s'en suit une première cuisson de la maiche au cours de laquelle on ajoute un peu d'eau au premier dépôt (dépôt 1), qui est chauffé jusqu'à $90{ }^{\circ} \mathrm{C}$ pendant environ 30 minutes. Cette opération permet de gélifier l'amidon dont les chaînes se déroulent et deviennent accessibles aux amylases conservées dans le surnageant. La marmite est retirée du feu et son contenu est ajouté au surnageant initialement recueilli. Le mélange se stabilise à une température de 60 à 70 ${ }^{\circ} \mathrm{C}$, favorable à l'activité des amylases qui hydrolysent l'amidon en des sucres simples (glucose, maltose, maltotriose, dextrines, etc.) ; ce mélange est laissé au repos pendant environ 12 heures pour une bonne saccharification et une décantation totale donnant le surnageant 2 et le dépôt 2 . Ensuite intervient une deuxième cuisson à 80 à $90{ }^{\circ} \mathrm{C}$ pendant 25 à 30 minutes, suivie d'une filtration pour séparer le drèche du filtrat ; le surnageant 2 est ajouté à ce filtrat et le mélange obtenu subit une troisième cuisson pour donner le moût de chakpalo. Le brassage est l'étape au cours de laquelle l'amidon et les protéines vont être hydrolysés en sucres fermentescibles et composés azotés par les enzymes (Alais et Linden, 1997). C'est aussi au cours de cette étape que sont observées les plus grandes variations dans les processus de production selon les groupes ethniques ou les régions.

La fermentation : le moût obtenu après brassage est filtré, quantifié, puis conditionné dans des gourdes habituellement utilisées pour la fermentation spontanée du chakpalo; ces gourdes contiennent déjà des souches naturelles de levures, du fait de leur utilisation antérieure. La fermentation dure 72 heures, à la température ambiante (entre 25 et $30^{\circ} \mathrm{C}$ ).

Matériel de production de chakpalo: le matériel utilisé pour la production artisanale du chakpalo se résume comme suit: on fait germer le sorgho dans des paniers et le malt vert est séché sur une bâche et broyé dans un moulin à maïs ; un foyer généralement en terre de barre ou à trois pierres sert à entretenir le feu de bois et le transfert de chaleur aux marmites métalliques de capacité variant entre 25 et 100 litres et utilisées pour la cuisson des produits intermédiaires et finis ; on emploie des bassines à fond perforé dans la filtration du moût, et divers récipients dans le dosage des matières premières et les autres opérations unitaires de la chaîne de production. Le moût est séparé du drèche à l'aide d'un filtre constitué d'une bassine ouverte à sa base et dont le fond est tapissé de brindilles recouvertes d'un morceau de sac de jute qui sert de toile filtrante. L'ensemble du dispositif est déposé sur un autre récipient qui recueille le filtrat. Une spatule est utilisée pour homogénéiser le moût au cours du chauffage afin d'éviter la calcination et l'incrustation des particules au fond de la marmite; des jarres et gourdes servent de cuve de fermentation et de récipients pour le conditionnement tandis que les calebasses sont utilisées pour la vente en détail du chakpalo.

Importance de l'activité de production de chakpalo: L'enquête a permis de recenser au total 459 productrices de chakpalo, à raison de $73 \%$ dans la commune de Glazoué et $27 \%$ dans la commune Dassa. La production de chakpalo est très développée dans la commune de Glazoué où elle constitue l'activité principale de $70 \%$ des productrices enquêtées et l'activité secondaire par rapport à la production agricole de $30 \%$ femmes. La production du chakpalo est une activité assurée exclusivement par les femmes; elles sont pour la plupart analphabètes (75\% des enquêtées) et appartiennent à la tranche d'âge comprise entre 16 et 65 ans, avec une moyenne d'âge de 46 ans. Elles sont exclusivement d'ethnie Idaatcha (100\% des productrices recensées) et ont une longue expérience dans le métier puisqu'elles commencent l'activité depuis leur adolescence en aidant leur mère ou leur tutrice. L'enquête a adressé essentiellement les productrices-vendeuses, mais il existe des productrices simples qui livrent le chakpalo à des revendeuses. On assiste aussi à une forme de production domestique non marchande, surtout pendant la saison sèche, destinée à l'autoconsommation, aux cérémonies, festivités et manifestations populaires. Une productrice de chakpalo transforme en moyenne $21,8 \mathrm{~kg}$ de sorgho par cycle de production à fréquence moyenne de 1,8 fois par semaine, soit en moyenne $39,2 \mathrm{~kg}$ de sorgho transformé par semaine. Un kilogramme de sorgho donne environ 2,5 litres de chakpalo. La quantité de sorgho transformée dans une année par les 459 productrices est estimée à 936,6 tonnes et équivaut à un volume total de $23414.5 \mathrm{hl}$ de chakpalo. Une typologie sommaire fondée sur le niveau de production répartit les productrices en trois catégories : les petites productrices qui transforment en moyenne $9 \mathrm{~kg}$ de sorgho par cycle de production et représentent 7,5\% des enquêtées; les moyennes productrices 


\section{Dossou et al. J. Appl. Biosci. Analyse économique de la production artisanale du chakpalo au Bénin : aspects sociaux et facteurs déterminants de la rentabilité financière de l'activité}

transformant en moyenne $21,8 \mathrm{~kg}$ de sorgho par cycle de production et représentent en moyenne $68 \%$ et les grandes productrices, qui transforment $36 \mathrm{~kg}$ de sorgho par cycle de production et représentent $24,5 \%$. Selon Pallier (1982), la bière du sorgho représente une part importante de l'artisanat alimentaire dans beaucoup de pays africains ; cet auteur a dénombré 600 dolotières qui produiraient environ 36 millions de litres de dolo par an dans la seule ville de Ouagadougou au Burkina Faso. A l'origine, la fabrication du dolo serait la spécialité de certaines ethnies de ce pays selon Broutin et Laura (1992), comme c'est le cas au Bénin. Dans la présente étude, l'enquête a révélé de nombreuses contraintes liées à la production du chakpalo et dont les plus importantes sont d'ordre énergétique, technologique et ergonomique. Sur le plan énergétique, la production du chakpalo est grande consommatrice de bois ; la technologie reste encore empirique avec du matériel primitif, impliquant une forte pénibilité des opérations unitaires. En ce qui concerne l'ergonomie, les productrices se plaignent souvent d'être exposées à la chaleur et la fumée et parfois à des cas de maladies telles que la tension artérielle, l'asthénie et les affections des voies respiratoires.

Consommation et appréciation du chakpalo: le chakpalo est souvent qualifié, à raison, de bière locale par les consommateurs puisque les études antérieures de Okoumanssoun (1984), Atègbo (1985) et Nago (1989) ont aussi montré que sa technologie s'apparente à celle de la bière industrielle à base d'orge. Parmi les nombreuses boissons et pâtes fermentées produites traditionnellement au Bénin, le chakpalo conserve une place de choix en raison principalement du nombre important de femmes qui se consacrent à sa production, à sa vente et sa large diffusion à travers le pays sous l'effet des flux migratoires (Atègbo, 1985; Nago, 1989). Contrairement à sa production, la consommation du chakpalo n'est pas l'apanage des peuples Idaatcha. II est vendu et consommé sur les places publiques, les marchés quotidiens et périodiques, les carrefours des voies publiques etc. C'est une boisson très appréciée de toutes catégories de personnes. Le succès relatif de la bière de sorgho auprès des consommateurs serait le fait de son prix relativement bas, des vertus thérapeutiques qui lui sont attribuées et de l'amélioration du régime alimentaire de millions de personnes à laquelle elle contribue (van der Aa Kühle et al., 2001). Les critères d'appréciation de la bière de chakpalo tels que décrits par les consommateurs sont: le goût, la couleur, le degré alcoolique et le taux d'acidité. Le goût et le degré alcoolique ont été identifiés par Kayodé et al. (2007) et Pale et al. (2010) comme les principaux critères de qualité utilisés par les consommateurs pour apprécier certaines bières traditionnelles (tchoukoutou, dolo). L'appréciation donnée par les consommateurs sur les critères relatifs au goût, à la couleur et au taux d'acidité ne varie pas significativement selon le genre $(P<0,05)$. Par contre pour le degré alcoolique, les femmes ont tendance à apprécier le chakpalo peu alcoolisé, tandis que les hommes préfèrent le chakpalo très alcoolisé. Des observations similaires ont été faites par Kayodé et al. (2007) sur le tchoukoutou. Ces auteurs ont remarqué qu'il y a une relation significative entre le genre et la fréquence de consommation du tchoukoutou. Le tchoukoutou est fréquemment ou régulièrement consommé par les hommes alors qu'il est occasionnellement ou rarement consommé par les femmes (Kayodé et al., 2007). Cette bière est considérée comme un stimulant lorsque les gens veulent exécuter un travail laborieux. Pour eux "tchoukoutou donne la force" (Van Liere, 1993). Cette caractéristique attribuée au tchoukoutou serait liée à son degré alcoolique et c'est probablement pour avoir les mêmes effets que les hommes préfèrent un chakpalo très alcoolisé. Selon les consommateurs, le bon chakpalo est de couleur brune, légèrement acide et alcoolisé. Ces critères d'appréciation du chakpalo permettent à certains consommateurs d'identifier parfois même la matière première utilisée (sorgho et/ou maïs). Le chakpalo fermenté préparé à base de maïs ou qui n'a pas subi une longue cuisson a une couleur généralement blanchâtre ou à peine brunâtre. Un chakpalo très acide n'est pas de bonne qualité ; il a soit séjourné longtemps avant sa mise en consommation ou ne provient pas de sorgho germé. Les productrices ont aussi ajouté que le chakpalo devient de plus en plus acide lorsque le ferment traditionnel utilisé est plusieurs fois recyclé. Lorsque la filtration du moût s'avère difficile et dure de longues heures, le filtrat peut s'acidifier et le chakpalo garde un goût acide très prononcé après la fermentation. Le degré alcoolique du chakpalo varie de 1,5 à $4,5 \%$ selon le niveau de fermentation. En général, celui des bières à base de sorgho serait compris entre 2 et $3 \%$ (Agu et Palmer, 1998 ; Briggs et al., 2004). La non maîtrise de la stabilité du chakpalo demeure l'une des contraintes majeures de la production par la méthode traditionnelle. Cette contrainte a été abordée par Konfo et al. (2014), qui ont démontré que l'adjonction de la poudre de Hemizygia bracteosa (Benth) au cours de la phase de 


\section{Dossou et al. J. Appl. Biosci. Analyse économique de la production artisanale du chakpalo au Bénin : aspects sociaux et facteurs déterminants de la rentabilité financière de l'activité}

l'empâtage engendre une augmentation significative de la stabilité avec $64 \%$ d'inhibition sur la flore aérobie mésophile totale, $100 \%$ d'inhibition sur les moisissures et $61 \%$ sur les coliformes.

Analyse économique de la production de chakpalo: l'analyse économique réalisée dans la présente étude se résume à la détermination de la marge brute de production du chakpalo. Elle s'est basée essentiellement sur les moyennes productrices mais a pris aussi en compte les autres catégories de productrices. Le résultat d'exploitation calculé à partir du produit de la vente du chakpalo et des charges totales de production est indiqué dans le Tableau 3.

\section{Charges de production}

Charges fixes et charges variables: il ressort des analyses que la valeur totale des charges d'amortissement est de 1124,97 FCFA (Tableau 2). L'approvisionnement en matière première (sorgho), le combustible (bois), la mouture du malt et la maind'œuvre de transformation représentent les charges variables dans la production du chakpalo. Les coûts de ces facteurs ont été calculés sur la base de 21,84 kg de sorgho transformé en chakpalo par production. Le malt de sorgho est moulu à 16,66 FCFA/kg.

Tableau 3 : Charges variables pour une capacité moyenne de transformation de 21,84 kg de sorgho

\begin{tabular}{llll}
\hline Paramètres & Quantité & Prix unitaire (FCFA) & Coût total (FCFA) \\
\hline Matière première (sorgho) & $21,84 \mathrm{Kg}$ & 111 & 2424,24 \\
Main d'œuvre & $3,06 \mathrm{femmes} /$ jour & 500 & 1530 \\
Achat de bois & $128,31 \mathrm{Kg}$ & 10 & 1283,1 \\
Mouture & $21,84 \mathrm{Kg}$ & 16,66 & 363,85 \\
\hline Total & & 5601,19 \\
\hline
\end{tabular}

Tableau 4 : Compte d'exploitation de la production du chakpalo

\begin{tabular}{llllc}
\hline Désignation & Unité & Quantité & Prix unitaire (FCFA) & Valeurs (FCFA) \\
\hline 1- Produit & & & & \\
Vente de chakpalo & $\mathrm{L}$ & 54,8 & 100 & 5480 \\
\hline Total 1: & & & & \\
\hline 2 Charges & & & & \\
\hline 2-1 Charges variables & $\mathrm{kg}$ & 21,84 & 111 & 2424,24 \\
Matières premières (Sorgho) & $\mathrm{F} / \mathrm{J}$ & 3,06 & 500 & 1530 \\
Main-d'œuvre & $\mathrm{kg}$ & 128,31 & 10 & 1283,1 \\
Achat de bois & $\mathrm{kg}$ & 21,84 & 16,66 & 363,85 \\
Coût de la mouture & & & & $\mathbf{5 6 0 1 , 1 9}$ \\
\hline Total 2-1: & & & & 57,69 \\
2-2 Charges fixes & & & 48,07 \\
Marmite & & & 288,46 \\
Foyer & & & $\mathbf{1 1 2 4 , 9 5}$ \\
Bassines & & & $\mathbf{6 7 2 6 , 1 4}$ \\
Petit matériel & & & $\mathbf{- 1 2 4 6 , 1 4}$ \\
\hline Total 2-2: & &
\end{tabular}

Matière première: la principale matière première utilisée dans la production du chakpalo est le sorgho.
Les sources d'approvisionnement en matières premières varient d'une productrice à l'autre et 


\section{Dossou et al. J. Appl. Biosci. Analyse économique de la production artisanale du chakpalo au Bénin : aspects sociaux et facteurs déterminants de la rentabilité financière de l'activité}

dépendent de la capacité de production, mais la plupart des productrices s'approvisionnent au marché de Glazoué où le sorgho est vendu à 111 FCFA par kilogramme au moment de l'étude et les 21,84 kg reviennent à 2424,24 FCFA.

Main-d'œuvre : une journée de travail est rémunérée à 500 FCFA dans la zone d'étude. Si toutes les opérations de fabrication du chakpalo sont exercées par une seule femme, la durée totale (TD) de travail est estimée à 12,66 h pour la transformation de $9 \mathrm{~kg}$ de sorgho, $24,5 \mathrm{~h}$ pour $21,84 \mathrm{~kg}$, et $39,2 \mathrm{~h}$ pour $50 \mathrm{~kg}$; ces durées correspondent respectivement à 1,58, 3,06 et 4,86 femme-jour (F/J).

Coût de l'énergie de cuisson : le tableau 3 présente les charges variables pour la capacité moyenne de transformation de 21,84 kg de sorgho par semaine. Le combustible utilisé dans la cuisson du chakpalo est le bois. La charrette de $400 \mathrm{~kg}$ est vendue à 4000 FCFA, soit $10 \mathrm{FCFA} / \mathrm{kg}$. On obtient 2,5 litres de chakpalo par $\mathrm{kg}$ de sorgho, soit un rendement de $250 \%$. Les mesures effectuées sur le terrain ont montré qu'il faut en moyenne $106 \mathrm{~kg}$ de bois pour préparer 45 litres de chakpalo, soit une consommation spécifique de 2,35 $\mathrm{kg} / \mathrm{l}$. Cette valeur est comparable à celles trouvées par Dossou et al. (2005a) et Nago (1995) qui indiquent respectivement une consommation spécifique de 2,69 $\mathrm{kg} / \mathrm{kg}$ et de 1,3 à 2,4 kg/kg pour la cuisson-séchage du gari. Le prix de revient $C$ de l'énergie consommée pour la transformation de 21,84 kg est estimé à 1283,1 FCFA (Tableau 3). L'une des principales contraintes évoquées par les productrices enquêtées est la consommation excessive de bois de chauffe. En effet, les trois niveaux de cuisson qu'exige la chaîne de production du chakpalo impliquent une importante consommation de bois et par conséquent, une augmentation considérable des charges variables. Le coût du combustible seul constitue environ $23 \%$ des charges variables de production. Zohoun (2003) et Dossou et al. (2005b) ont observé que le coût du combustible (bois de chauffe) a eu une incidence négative sur le résultat d'exploitation de la production du gari au sud du Bénin. Dans les localités de forte production de chakpalo, le bois de chauffe devient déjà de plus en plus rare et très coûteux et la pression exercée par les productrices sur le couvert végétal provoque la déforestation et menace l'équilibre de l'écosystème.

Résultat d'exploitation de la production de chakpalo : le compte d'exploitation de la production du chakpalo réalisé pour une capacité moyenne de transformation de $21,84 \mathrm{~kg}$ de sorgho (soit environ 54 litres de chakpalo), est présenté dans le tableau 4. Pour cette capacité moyenne de production, l'activité dégage une marge brute négative de -1246,14 FCFA. Ce résultat tient du faible niveau de production et des charges variables élevées $(6726,14$ FCFA). II faut atteindre un seuil de transformation de $50 \mathrm{~kg}$ de sorgho avant de prétendre à une marge brute positive de 395,45 FCFA. Des études similaires réalisées par Kayodé et al. (2007) sur le tchoukoutou, ont montré que l'activité de production du tchoukoutou dégage une marge nette comprise entre 2365 et 17212 FCFA par mois et que les bénéfices dépendent du rendement en bière et de la quantité de matière première transformée. Ces mêmes auteurs ont remarqué que les productrices avec des rendements en bière les plus élevés n'ont pas les bénéfices les plus élevés dû à une excessive dilution de la bière entraînant ainsi de faibles prix de vente de la bière dont seulement les $80 \%$ peuvent être vendus (Kayodé et al., 2007). Les marges bénéficiaires élevées observés au niveau de la production du tchoukoutou s'expliqueraient notamment par le niveau de production (100 à 122,8 kg de matière première transformée) et le coût de la matière première $(65,15$ à $88 \mathrm{FCFA} / \mathrm{kg})$. Dans les localités enquêtées en particulier et pour l'ensemble du secteur de l'artisanat alimentaire en général, les productrices traditionnelles n'intègrent pas dans leur calcul de marge bénéficiaire ni la main-d'œuvre salariée, ni le coût du bois de chauffe. Pourtant, ces facteurs de production s'évaluent respectivement à $27 \%$ et $23 \%$, soit en somme à $50 \%$ des charges totales de production. En éliminant ces deux paramètres, on obtient une marge brute apparente positive. C'est donc la principale raison qui amène les productrices à maintenir l'activité malgré les résultats financiers décevants. Mais au-delà de la rentabilité économique, la production du chakpalo assure des fonctions sociales inestimables : elle crée des emplois durables, conforte le statut social de la productrice, fournit la boisson locale pour les cérémonies rituelles et les fêtes traditionnelles et entretient un circuit de commercialisation bien établi dans les communautés. Les mêmes observations ont été faites Kayodé et al. (2007) et Lyumugabe et al. (2010). En effet, pour ces auteurs, la bière de sorgho est une boisson ancestrale généralement utilisée lors de diverses cérémonies africaines tels que les mariages, les dots, les cérémonies funéraires, le festival populaire et annuel du sorgho, les cérémonies de sortie d'enfant etc. (Kayodé et al., 2007 ; Lyumugabe et al., 2010). Pallier (1982) quant à lui observe que si le rôle économique du dolo est faible, 


\section{Dossou et al. J. Appl. Biosci. Analyse économique de la production artisanale du chakpalo au Bénin : aspects sociaux et facteurs déterminants de la rentabilité financière de l'activité}

son rôle social reste cependant très important au Mali et au Burkina Faso, parce que le cabaret est un lieu de rencontre et de discussions. Des observations semblables ont été faites par l'ONASA (1990) qui a mis en cause la rentabilité économique du gari produit au Bénin, comparativement à celui du Nigéria et du Togo. En confirmant que ce produit n'est pas compétitif, Adjovi (2002) estime que l'une des justifications de la poursuite de cette activité, malgré des résultats d'exploitation peu incitatifs, tiendrait non seulement du souci de conserver la matière première par la transformation mais surtout du caractère social

\section{CONCLUSION}

L'étude a permis de diagnostiquer les coûts afférant à la production artisanale du chakpalo dans les localités d'origine de cette boisson traditionnelle au Bénin. En dehors de la forte pénibilité du travail et de nombreuses autres contraintes relatives prioritairement à la longue durée des opérations et au caractère rudimentaire des équipements utilisés dans cette activité, la main d'œuvre et le combustible consommé engagent 50\% des coûts de production du chakpalo. Du reste, l'analyse économique a révélé que l'activité n'est pas rentable puisque le résultat d'exploitation est négatif et s'évalue à -1246,14 FCFA pour la capacité moyenne

\section{RÉFÉRENCES BIBLIOGRAPHIQUES}

Adjovi AN, 2002. Identification des potentialités agricoles au Bénin par l'estimation des couts des ressources domestiques. Rapport d'études; document de travail $n^{\circ} / 2001$; Cellule d'Analyse de la Politique Économique (CAPE), Cotonou, Bénin.

Agu RC. and Palmer GH, 1998. A reassessment of sorghum for lager-beer brewing. Bioresearch Technology. 66, 253-261.

Alais C and Linden G, 1997. Abrégé de biochimie alimentaire. 4 e éd. Paris: Masson, 160-165.

Atègbo $E A D, 1985$. Contribution à l'étude biochimique et technologique du chakpalo. Influence de quelques paramètres sur la qualité et l'aptitude à la conservation de cette boisson. Thèse d'ingénieur agronome, Faculté des Sciences Agronomique (FSA), Université d'AbomeyCalavi, Abomey-Calavi, 83p,.

Ballogou VY, Dossou J, de Souza CA, 2011. Controlled Drying Effect on the Quality of Sorghum Malts Used for the Chakpalo Production in Benin. Food and Nutrition Sciences. 2, 156-161.

Bhise VJ, Chavan JK, Kadam SS, 1988. Effects of déterminant du produit. Mais la faible performance des technologies mises en œuvre dans le secteur de l'artisanat alimentaire est aussi à l'origine de la faible compétitivité des produits. C'est pourquoi nos études en cours sur le chakpalo se concentrent désormais sur l'amélioration de la technologie et de la qualité hygiénique du produit, l'augmentation de la capacité de production des unités traditionnelles, la création et la viabilisation de malteries et brasseries modernes à petite et moyenne échelle dans les localités d'origine du chakpalo.

de transformation de $21,84 \mathrm{~kg}$ de sorgho; ce résultat concerne plus de $75 \%$ de productrices de chakpalo. Pourtant, cette activité mobilise beaucoup de femmes pour qui elle constitue l'unique source de revenu. II est donc impératif de mener des études pour améliorer la technologie de production, les équipements utilisés ainsi que la qualité du produit afin d'augmenter la rentabilité de l'activité. Ceci constituerait une avancée notable dans la lutte contre la pauvreté et l'amélioration des conditions de vie des femmes des localités enquêtées.

malting on proximate composition and in vitro protein digestibility of grain sorghum. Journal of Food Sciences and Technology 25, 327-329.

Briggs DE, Bolton CA, Brookes PA, Stevens R, 2004. Native African Beers. In Brewing Science and Practice ed., pp. 589-605. Boca Raton, Cambridge: CRC Press, Woodhead Publishing Ltd.

Broutin C et Laura P, 1992. Artisanat alimentaire et consommation de bois de feu; Rapport d'étude; Association Bois de Feu; Réseau TPA/GRET; Paris, France ;

Dossou J, Egounlety M, Zohoun E, N'Zamdjo G, 2005a. Production du gari commercial: options énergétiques et caractéristiques physico-chimiques et sensorielles du produit. Journal de la Recherche Scientifique. Université Lomé (Togo), Série A, 7I 29-36

Dossou J, Egounlety M, Zohoun E, N'Zamjo G, 2005b. Analyse de l'incidence du coût de l'énergie de cuisson sur la marge brute du gari produit au sud du Bénin. Journal de la Recherche 


\section{Dossou et al. J. Appl. Biosci. Analyse économique de la production artisanale du chakpalo au Bénin : aspects sociaux et facteurs déterminants de la rentabilité financière de l'activité}

Scientifique. Université. Lomé (Togo), série A, 7 (1) : 49-58.

Dossou J, Ballogou V, de Souza C, 2011. Etude de la dynamique microbienne et la qualité du chakpalo fermenté à la levure commerciale (Saccharomyces cerevisiae) et au ferment traditionnel et stabilisé par la pasteurisation. Journal de la Recherche Scientifique. Université. Lomé (Togo), série A, $13:$ 39-51.

Fabre $P, 1994$. Note méthodologique générale sur l'analyse de filières pour l'analyse économique et politique. Document de formation pour la planification agricole. FAO, Rome $105 \mathrm{p}$.

FAO, 1985. Les femmes dans l'agriculture. Food and Agriculture Organization of United Nation. Rome (I). Le courrier. 91, 52-55.

Kayodé APP, Adegbidi A, Hounhouigan JD, Linnemann AR, Nout MJR, 2005. Quality of farmers' varieties of sorghum and derived foods as perceived by consumers in Benin. Ecology of food and Nutrition, 44, 271-294.

Kayodé APP, Hounhouigan DJ, Nout MJR, Niehof A, 2007. Household production of sorghum beer in Benin: Technological and socio-economical aspects. International Journal of Consumer Studies, 31, 258-264.

Konfo CTR, Chabi NW, Agbadjizo J, DahouenonAhoussi E, Soumanou MM, Sohounhloué DCK, 2014. Influence de la feuille de Hemizygia bracteosa (Benth) sur la qualité de la bière du sorgho "tchakpalo" produite au Bénin. International Journal of Innovation and Applied Studies. 7, 2, 453-463.

Lyumugabe L, Kamaliza G, Bajyana E, Thonart Ph, 2010. Microbiological and physico-chemical characteristics of Rwandese traditional beer "Ikigage". African Journal of Biotechnology. 9, 4241-4246.

Nago CM, 1995. La préparation artisanale du gari au Bénin: aspects technologiques et physicochimiques ; in Transformation alimentaire du manioc ; ORSTOM éd. ; France ; pp. 475-493;

Nago MC, 1989. Technologies traditionnelles et alimentation au Bénin: Aspects techniques, biochimiques et nutritionnels: application à quelques aliments fermentés locaux. Etude de la technologie traditionnelle de préparation du "Chakpalo". Document technique. Université Nationale du Bénin.

Office National de Sécurité Alimentaire (ONASA), 1990. Production et commercialisation du gari au Bénin ; Vol. 5, $74 \mathrm{p}$.

Okoumassoun EL, 1984. Le chakpalo: Aspects Technologiques et analyse qualitative des glucides par chromatographie sur couche mince. Mémoire de fin d'étude DET, Université Nationale du Bénin, Cotonou.

Osseyi E.G. Tagba P Karou S.D., Ketevi A.P., Lamboni C.R ,et al., 2011. Stabilization of the traditional sorghum beer, "tchoukoutou" using rustic wine-making method. Advanced Journal of Food Sciences and Technology. 3, 254-258.

Pale S, Taonda SJB, Bougouma B, Mason SC, 2010. Sorghum malt and traditional beer (Dolo) quality assessment in Burkina Faso. Ecology of Food and Nutrition. 49, 129-141.

Pallier G, 1982. Les dolotières à Ouagadougou (Haute Volta), in La croissance de pays tropicaux : dix études sur l'approvisionnement des villes; CEGET; Bordeaux, France ;

Traoré T Mouquet C, Icard-Vernière C, Traoré A S, Trèche S., 2004. Changes in nutrient composition, phytate and cyanide contents and a-amylase activity during cereal malting in small production units in Ouagadougou (Burkina Faso). Food Chemistry. 88, 105-114.

Van der Aa Kühle A, Mouquet $C$, Icard-Vernière $C$, Traoré A S, Trèche S2001. Identification and characterization of Saccharomyces cerevisiae strains isolated from West African sorghum beer. Yeast. 18, 1069-1079.

Van Liere MJ, 1993. Coping with Household Food Insecurity: a Longitudinal and Seasonal Study Among the Otammari in North-western Benin. Wageningen University, Wageningen.

Zohoun E, 2003. Production du gari commercial option énergétique et caractéristiques physicochimiques et sensorielle du produit. Thèse d'ingénieur agronome. Faculté des Sciences Agronomiques de l'Université d'AbomeyCalavi, Bénin. 